\title{
Charge Transport in Azobenzene-Based Single-Molecule Junctions
}

\author{
Youngsang Kim, ${ }^{1, *}$ Aran Garcia-Lekue, ${ }^{2,3}$ Dmytro Sysoiev, ${ }^{4}$ Thomas Frederiksen, ${ }^{2,3}$ Ulrich Groth, ${ }^{4}$ and Elke Scheer ${ }^{1, \dagger}$ \\ ${ }^{1}$ Department of Physics, University of Konstanz, 78457 Konstanz, Germany \\ ${ }^{2}$ Donostia International Physics Center (DIPC)-UPV/EHU, Paseo Manuel de Lardizabal 4, 20018 San Sebastian, Spain \\ ${ }^{3}$ IKERBASQUE, Basque Foundation for Science, E-48011 Bilbao, Spain \\ ${ }^{4}$ Department of Chemistry, University of Konstanz, 78457 Konstanz, Germany
}

(Received 28 June 2012; published 28 November 2012)

\begin{abstract}
Azobenzene-derivative molecules change their conformation as a result of a cis-trans transition when exposed to ultraviolet or visible light irradiation and this is expected to induce a significant variation in the conductance of molecular devices. Despite extensive investigations carried out on this type of molecule, a detailed understanding of the charge transport for the two isomers is still lacking. We report a combined experimental and theoretical analysis of electron transport through azobenzene-derivative single-molecule break junctions with Au electrodes. Current-voltage and inelastic electron tunneling spectroscopy (IETS) measurements performed at $4.2 \mathrm{~K}$ are interpreted based on first-principles calculations of electron transmission and IETS spectra. This qualitative study unravels the origin of a slightly higher conductance of junctions with the cis isomer and demonstrates that IETS spectra of cis and trans forms show distinct vibrational fingerprints that can be used for identifying the isomer.
\end{abstract}

DOI: 10.1103/PhysRevLett.109.226801

Photochromic molecules are promising building blocks for the realization of functional molecular circuits [1,2]. In recent years, the charge transport in these molecules has been extensively investigated for a wide range of systems [3-9]. When photochromic molecules in solution are exposed to radiation of ultraviolet or visible light, the molecules undergo a reversible conformational change. In the case of azobenzene and its derivatives, it is a cistrans transition going along with a pronounced change of the geometry. In addition, upon this isomerization the electronic properties change markedly, providing an opportunity to build photoactive devices such as optically driven electronic switches or memories, if these molecules are bridging nanoelectrodes.

The azobenzene class of molecules has become an archetype of molecular photoswitch research, due to their simple structure and the significant difference of the electronic system between their cis and trans isomers [1,4,10-13]. The dynamics of the switching process of azobenzene chemisorbed on surfaces has also been studied in detail [14]. However, the charge transport properties of both isomers, when embedded in a junction with electrodes are still ambiguous. Devices with the cis isomer have been reported to exhibit a higher $[4,5,13,15]$ or lower $[1,10]$ conductance than the trans isomer. The difference in conductance between the two conformations may arise from a shift of the energy of the current-carrying molecular level $[4,10]$ or by the change of tunneling barrier width due to the length change going along with the isomerization $[5,13,15]$. In order to clarify these issues, transport measurements using single-molecule junctions (SMJs) at low temperature, including inelastic electron tunneling spectroscopy (IETS), are required. IETS allows us to detect vibrational
PACS numbers: 73.63. $-\mathrm{b}, 61.46 .-\mathrm{w}, 71.15 .-\mathrm{m}, 81.07 . \mathrm{Nb}$

excitations in nanoscale junctions, and is very sensitive to the molecular conformation, contact geometry, and electrode-molecule coupling [16-22]. Hence, IETS spectra provide evidence of the cis-trans conformation, as well as information on the inelastic transport processes across different isomers. In addition, the analysis of current-voltage $(I-V)$ characteristics based on a single-level transport model and $a b$ initio quantum chemical calculations shed light on the character of the dominant molecular energy level and on the contact coupling in the two different isomers $[2,19,23]$.

In this Letter, we investigate charge transport properties through single Azobenzene-ThioMethyl (AzoTM) molecules in a mechanically controlled break-junction (MCBJ) system at $4.2 \mathrm{~K}$. Single-molecule conductance, $I-V$ characteristics, and IETS spectra of molecular junctions are measured and compared with first-principles transport calculations. These studies elucidate the charge transport properties of single azobenzene-derivative molecules.

The azobenzene-derivative molecules containing $\mathrm{CH}_{2}$ side chains with sulfur (S) end groups are newly developed to control the coupling strength between the switching kernel and the metal electrodes. It has been shown that the switching process might be suppressed when the coupling of the switching core to gold electrodes is too strong $[11,12]$. Furthermore, side chains are necessary that bear enough flexibility for the molecule to adopt to the electrode distance in both isomers without breaking the contact to the metal electrodes.

Either cis or trans isomers, initialized by irradiating UV or visible light, respectively, are assembled on the lithographically defined $\mathrm{Au}$ electrodes and then mounted in a custom-designed cryostat equipped with a MCBJ system. The optical properties, synthesis method of the molecules, 

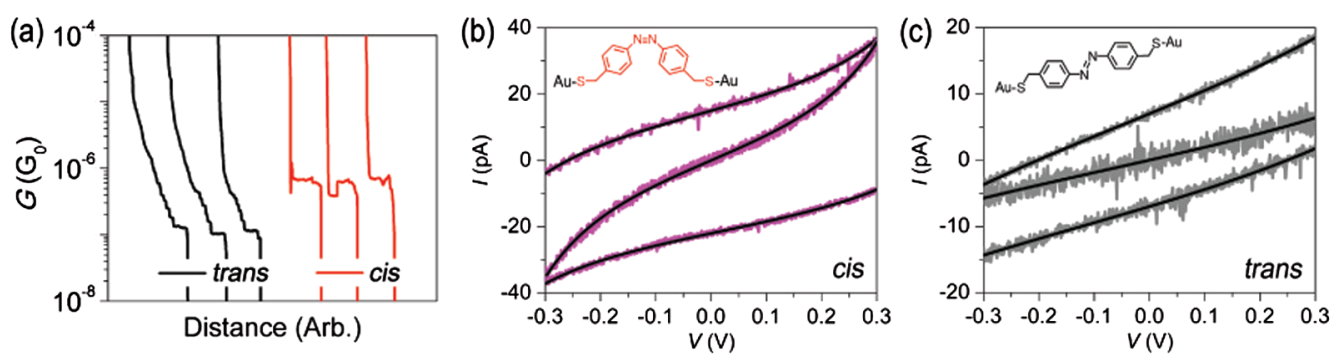

FIG. 1 (color online). (a) Conductance traces of cis and trans isomers of AzoTM SMJs are obtained by applying $70 \mathrm{mV}$ of dc voltage. The $I-V$ curves of (b) cis and (c) trans isomers with fitting curves (black solid line). The curves are vertically offset for enhancing clarity. The single-level model fitting parameters are (from top to bottom) for cis (b) $\Gamma=0.21 \mathrm{meV},\left|E_{0}\right|=0.27 \mathrm{eV}$; $\Gamma=0.24 \mathrm{meV},\left|E_{0}\right|=0.24 \mathrm{eV} ; \Gamma=0.21 \mathrm{meV},\left|E_{0}\right|=0.30 \mathrm{eV}$ and for trans (c) $\Gamma=0.22 \mathrm{meV},\left|E_{0}\right|=0.55 \mathrm{eV} ; \Gamma=0.31 \mathrm{meV}$, $\left|E_{0}\right|=0.62 \mathrm{eV} ; \Gamma=0.34 \mathrm{meV},\left|E_{0}\right|=0.59 \mathrm{eV}$. In the inset of (b) and (c), the structures of AzoTM SMJs are presented.

and the device fabrication are described in the Supplemental Material [24]. In order to determine the characteristic conductance values of the Au-AzoTM-Au junctions, the junctions were repeatedly opened and closed while recording the conductance. Figure 1(a) presents typical conductance traces acquired during opening processes for both isomers. Clearly, cis isomers show a larger conductance value than trans isomers. The averaged values of the conductance for cis and trans isomers are $(4.9 \pm 3.4) \times 10^{-7} \mathrm{G}_{0}$ and $(1.6 \pm 0.7) \times 10^{-7} \mathrm{G}_{0}$, respectively, (conductance quantum $G_{0}=2 e^{2} / h$ ), as obtained by fitting the conductance histograms shown in the Supplemental Material [24]. This relatively small difference between cis and trans and low conductance is attributed to the existence of $\mathrm{CH}_{2}$ side chains acting as tunneling barriers and thus reducing the voltage drop across the azobenzene core [25].

With the aim of clarifying the transport properties of single AzoTM molecules, the $I-V$ characteristics were measured at the lowest conductance regime for cis and trans isomers as shown in Figs. 1(b) and 1(c). The $I-V$ s were measured between $4.2 \mathrm{~K}$ and $40 \mathrm{~K}$, and were found to be temperature independent, signaling off-resonant transport [24]. These $I-V$ curves were analyzed and fitted using the single-level model $[2,19,23]$. This model assumes a single molecular orbital at energy $E_{0}$ coupled symmetrically to each lead via the coupling constant $\Gamma$ (see Supplemental Material) [24]. This yields a resonance with Lorentzian shape in the transmission function $T(E)=\Gamma^{2} /[(E-$ $\left.\left.E_{0}\right)^{2}+\Gamma^{2}\right]$. The current is calculated as an integration over the bias window using the Landauer formula [24]. The values of $\Gamma$ and $\left|E_{0}\right|$ extracted from the measured $I-V$ curves are listed in Table. I. The $\Gamma$ values are small and do not change markedly between the isomers, because they are dominated by the alkane chains. The difference in conductance is thus fully caused by a shift of the molecular orbital.

In order to support our experimental analysis and to obtain a deeper physical understanding of the observed conductance characteristics, the transmission spectra for $\mathrm{Au}-\mathrm{AzoTM}-\mathrm{Au}$ junctions were calculated using density functional theory (DFT) combined with nonequilibrium
Green's function (NEGF) methods. The electronic structure and geometries are calculated with the SIESTA code [26], and the elastic transport is simulated for the TRANSIESTA setup [27]. We use a supercell description of the scattering region with an AzoTM molecule sandwiched between $\mathrm{Au}$ electrodes made up of $6 \mathrm{Au}(111)$ layers. A 5-atom pyramidlike conformation of the Au atoms is assumed in the contact. The generalized gradient approximation (GGA) was employed for exchange correlation [28]. Further details of the calculations are given in the Supplemental Material [24].

In Fig. 2 we represent the computed transmission curves obtained for varying electrode separations $L$ (measured between the $\mathrm{Au}$ atoms binding to the molecule). We observe that cis and trans isomers exhibit distinct features similar to what was recently reported for monolayers of azobenzene connected to low-coordinated Au atoms [29]. For the cis [Fig. 2(a)] isomer, regardless of the stretching exerted on the molecule, the charge transport around the Fermi level is mediated by the tail of a resonance delocalized over the entire molecule. In the case of trans isomers, a double-peak feature is observed for each electrodeelectrode separation, which we trace back to the interplay between two molecular orbitals. One of the orbitals of the trans isomer extends across the whole molecule whereas the other orbital is very localized around the $\mathrm{N}=\mathrm{N}$ bond region. A weak coupling between these two orbitals lying close in energy gives rise to a wide peak and a Fano-like resonance. We note that while the energetic position of the localized state may suffer from deficiencies of GGA [30], it

TABLE I. Averaged fitting parameters, $\Gamma$ and $\left|E_{0}\right|$, of the single-level model for $c i s$ and trans isomers, as obtained from the experimentally measured thirteen individual $I-V \mathrm{~s}$. The conductance $G$ (in units of $G_{0}$ ) values are obtained from the conductance histogram.

\begin{tabular}{lccc}
\hline \hline & $\Gamma(\mathrm{eV})\left(\times 10^{-4}\right)$ & $\left|E_{0}\right|(\mathrm{eV})\left(\times 10^{-1}\right)$ & $G\left(G_{0}\right)\left(\times 10^{-7}\right)$ \\
\hline cis & $2.8 \pm 1.0$ & $3.6 \pm 0.5$ & $4.9 \pm 3.4$ \\
trans & $2.6 \pm 0.6$ & $5.2 \pm 0.6$ & $1.6 \pm 0.7$ \\
\hline \hline
\end{tabular}



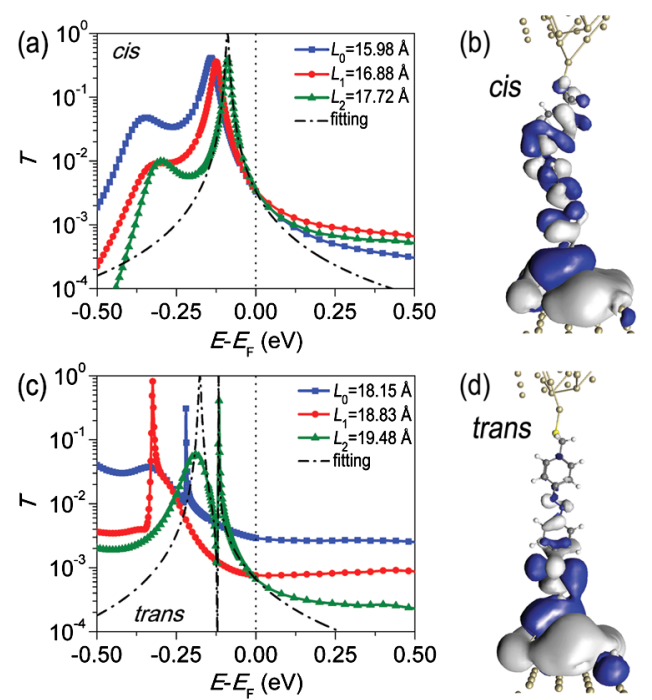

FIG. 2 (color online). Transmission curves for (a) cis and (c) trans isomers for different bridging distances. The dash-dotted lines in (a) and (c) indicate the representative single-level model and Fano resonance fits for cis and trans, respectively. The corresponding dominant transmission eigenchannels, i.e., isosurface of the scattering state incoming from the bottom side, for (b) cis $\left(L_{2}=17.72 \AA\right)$ and (d) trans $\left(L_{2}=19.48 \AA\right)$ isomers are shown.

has a negligible contribution to the transmission at the Fermi level. Consequently, the charge transport for the trans isomer is also dominated by the delocalized molecular orbital. These results suggest that the conductance for the cis isomer is higher than the trans isomer because the current-carrying molecular state is closer to the Fermi energy.

For the cis [Fig. 2(c)] isomer, regardless of the stretching exerted on the molecule, the charge transport around the Fermi level is mediated by the tail of a resonance related to the highest occupied molecular orbital (HOMO). In the case of trans isomers, a double-peak feature is observed for each electrode-electrode separation, which we trace back to the interplay between the HOMO-1 and HOMO molecular orbitals. The HOMO-1 orbital of the trans isomer extends across the whole molecule, whereas the HOMO orbital is very localized around the $\mathrm{N}=\mathrm{N}$ bond region. A weak coupling between these two orbitals lying close in energy gives rise to a wide peak and a Fano-like resonance. The latter has a negligible contribution to the transmission at the Fermi level and, consequently, the charge transport around the Fermi level is dominated by the HOMO-1 molecular orbital. Accordingly, the transport level is further away from the Fermi level than in the case of the cis isomer. These results suggest that the difference in the conductance of the isomers is due to the difference of their corresponding resonant states, in agreement with the experimental findings.

The resonances in the transmission of $c i s$ isomers can be fitted using the single-level model, as shown for a chosen electrode-electrode separation in Fig. 2(a) (dash-dotted line). For the trans isomers, the transmission can be fitted using the Fano resonance model described in the Supplemental Material [24]. The fitting for one bridging distance is given in Fig. 2(c) (dash-dotted line). In both cistrans cases we get values for the energy of the dominant resonant level, closer to Fermi level than the values extracted from experimental $I-V$ curves, which might be due to the underestimation of the HOMO-LUMO gap in DFT [31]. The single-level and Fano resonance models considered here, assume symmetric coupling to the electrodes. Overall, the fitting of the calculated transmission curves is very good, and the small deviations observed in Figs. 2(a) and 2(c) may be ascribed to a low degree of asymmetry in the systems. Numerical values of the fitting parameters are given in the Supplemental Material [24].

In the right panels of Fig. 2 the scattering states corresponding to the most transmitting eigenchannel (incoming waves from the bottom side) are visualized for the cis [in Fig. 2(b)] and trans [in Fig. 2(d)] isomers [32]. It is seen that the eigenchannel for cis decays more slowly through the molecular region as for trans, which reveals a bottleneck in the central region of the molecule around the $\mathrm{N}=\mathrm{N}$ bond. This is consistent with the larger conductance found numerically for the cis isomer. Our calculations also reveal that the conductance of the cis isomer [in Fig. 2(a)] is almost independent of the electrode-electrode separation while for the trans isomer [in Fig. 2(c)] the conductance markedly decreases upon increasing the bridging distance, which is consistent with what is observed in the experimental stretching curves of Fig. 1(a). While our simulations reproduce qualitatively the experimental trends, the calculated conductance values are, however, significantly larger than the experimental ones. This discrepancy may be due to the fact that DFT-GGA is known to underestimate the HOMOLUMO gap, and as a result, conductance values at Fermi level obtained with DFT can be significantly larger than those obtained with methods which better describe the HOMOLUMO gap, such as GW method[31,33]. Besides, the discrepancy might also be related to qualitatively different $\mathrm{Au}-\mathrm{S}$ bonding motifs between theory and experiment [34].

Further support for the molecules being in the predefined conformation, i.e., cis or trans, can be obtained by studying differences in IETS for the two isomers. To date, several IETS studies have been performed for test bed molecules such as alkane and benzene [17,19-21]. However, IETS measurements on photoswitching molecules have not been reported so far. Figure 3(a) shows experimental IETS spectra of Au-AzoTM-Au junctions for both isomers, measured using a standard lock-in technique $[19,20]$ and by averaging over several measurements [24]. IETS for cis and trans isomers exhibit distinct features, especially in voltage range 120-200 meV. IETS for trans isomers shows a strong signal at $\sim 160 \mathrm{meV}$, while cis isomers present a dominant peak at $\sim 190 \mathrm{meV}$. These variations in the IETS 

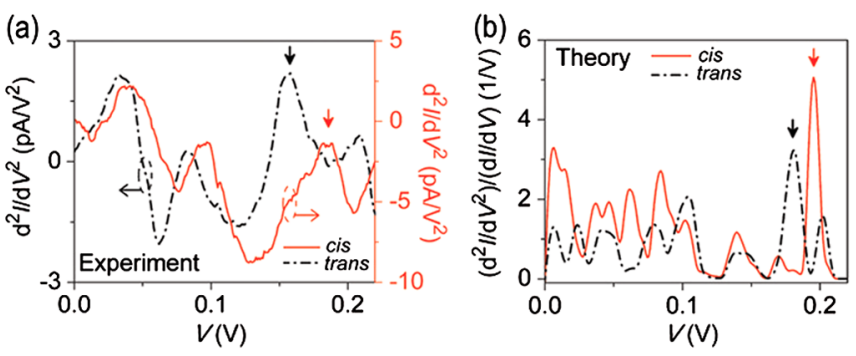

FIG. 3 (color online). (a) Experimental and (b) theoretical symmetrized IETS spectra for cis and trans isomers (averaged over several configurations) at temperature $T=4.2 \mathrm{~K}$, and $6 \mathrm{meV}$ for experimental and $5 \mathrm{meV}$ for theoretical ac modulation, respectively.

spectra reveal that the molecules were isomerized in different forms. Consequently, IETS spectra may be used as fingerprints for the differentiation of cis-trans isomers of the AzoTM molecule [24].

With the aim of elucidating the origin of these differences in the IETS, we have simulated the inelastic corrections to the tunneling current using the INELASTICA package $[24,35,36]$. The simulated IETS spectra are shown in Fig. 3(b), as obtained by averaging IETS for three different stretching conditions and neglecting asymmetric contributions [24]. Direct comparison with experiments requires detailed insight into the atomic arrangement of the junction. As this is largely unknown in MCBJ, it can explain certain differences between theory and experiment in Fig. 3, in particular in the low-bias region. However, robust features of both experiment and theory are observed in the voltage range $120-200 \mathrm{meV}$, where the cis (trans) isomer shows a distinct inelastic signal around $\sim 200 \mathrm{meV}$ $(\sim 180 \mathrm{meV})$ as indicated by arrows. Looking at the underlying vibrational modes giving rise to these signals we conclude that the peaks originate from modes with a strong $\mathrm{N}=\mathrm{N}$ stretching character [24]. Indeed the atomic arrangement around the $\mathrm{N}=\mathrm{N}$ bond is the most distinctive one between the cis-trans isomers and, as a result, the vibrational energies for modes involving motion of these atoms may be different. Since some of these modes are IETS active, distinguishable IETS signals are to be expected. This analysis supports that the observed differences in the experimental IETS are indeed caused by the cis-trans conformation.

In conclusion, we have investigated both experimentally and theoretically the elastic and inelastic charge transport properties of single azobenzene-based molecular devices. Our results demonstrate that the current-carrying energy level of trans isomer is further away from the Fermi energy than cis, resulting in lower conductance by roughly a factor of three. While the conductance ratio between cis and trans has a reasonable value, the overall conductance of these junctions is probably smaller than desired for applications in real devices. However, we have shown that the cis-trans isomers possess unambiguously different IETS fingerprints which thus enable an alternative readout path for identifying the state of azobenzene-based molecular switches.

The work in Konstanz was supported by the DFG through SFB767, the Krupp Foundation, and the Center for Applied Photonics. A. G.-L. and T.F. acknowledge funding from the Spanish Ministerio de Ciencia e Innovación (Grant No. FIS2010-19609-C02-01), the ETORTEK program funded by the Basque Departamento de Industria, and the Diputación Foral de Guipuzcoa. Y. K. and A. G.-L. contributed equally to this work.

*Present address: Department of Mechanical Engineering, University of Michigan, Ann Arbor, MI-48109, USA.

${ }^{\dagger}$ Corresponding author. elke.scheer@uni-konstanz.de

[1] M. D. Valle, R. Gutierrez, C. Tejedor, and G. Cuniberti, Nat. Nanotechnol. 2, 176 (2007).

[2] J.C. Cuevas and E. Scheer, Molecular Electronics: An Introduction to Theory and Experiment (World Scientific Publishers, Singapore, 2010), 1st ed.

[3] S. Yasuda, T. Nakamura, M. Matsumoto, and $\mathrm{H}$. Shigekawa, J. Am. Chem. Soc. 125, 16430 (2003).

[4] K. Smaali, S. Lenfant, S. Karpe, M. Oçafrain, P. Blanchard, D. Deresmes, S. Godey, A. Rochefort, J. Roncali, and D. Vuillame, ACS Nano 4, 2411 (2010).

[5] J. M. Mativetsky, G. Pace, M. Elbing, M. A. Rampi, M. Mayor, and P. Samori, J. Am. Chem. Soc. 130, 9192 (2008).

[6] S. J. van der Molen, J. Liao, T. Kudernac, J. S. Agustsson, L. Bernard, M. Calame, B. J. van Wees, B. L. Feringa, and C. Schönenberger, Nano Lett. 9, 76 (2009).

[7] A. J. Kronemeijer, H. B. Akkerman, T. Kudernac, B. J. van Wees, B. L. Feringa, P. W. M. Blom, and B. de Boer, Adv. Mater. 20, 1467 (2008).

[8] A.C. Whalley, M.L. Steigerwald, X. Guo, and C. Nuckolls, J. Am. Chem. Soc. 129, 12590 (2007).

[9] S. V. Aradhya, J. S. Meisner, M. Krikorian, S. Ahn, R. Parameswaran, M. L. Steigerwald, C. Nuckolls, and L. Venkataraman, Nano Lett. 12, 1643 (2012).

[10] C. Zhang, M.-H. Du, H.-P. Cheng, X.-G. Zhang, A.E. Roitberg, and J. L. Krause, Phys. Rev. Lett. 92, 158301 (2004).

[11] A. S. Kumar, T. Ye, T. Takami, B.-C. Yu, A. K. Flatt, J. M. Tour, and P. S. Weiss, Nano Lett. 8, 1644 (2008).

[12] M. J. Comstock, N. Levy, A. Kirakosian, J. Cho, F. Lauterwasser, J.H. Harvey, D. A. Strubbe, J. M. J. Fréchet, D. Trauner, S. G. Louie et al., Phys. Rev. Lett. 99, 038301 (2007).

[13] S. Martin, W. Haiss, S. J. Higgins, and R. J. Nichols, Nano Lett. 10, 2019 (2010).

[14] C. Bronner, M. Schulze, S. Hagen, and P. Tegeder, New J. Phys. 14, 043023 (2012).

[15] Y. Kim, G. Wang, M. Choe, J. Kim, S. Lee, S. Park, D.-Y. Kim, B. H. Lee, and T. Lee, Org. Electron. 12, 2144 (2011).

[16] A. Troisi and M. A. Ratner, Nano Lett. 6, 1784 (2006).

[17] H. Song, Y. Kim, Y.-H. Jang, H. Jeong, M. A. Reed, and T. Lee, Nature (London) 462, 1039 (2009). 
[18] M. Paulsson, C. Krag, T. Frederiksen, and M. Brandbyge, Nano Lett. 9, 117 (2009).

[19] Y. Kim, T. Pietsch, A. Erbe, W. Belzig, and E. Scheer, Nano Lett. 11, 3734 (2011).

[20] Y. Kim, H. Song, F. Strigl, H. F. Pernau, T. Lee, and E. Scheer, Phys. Rev. Lett. 106, 196804 (2011).

[21] J. G. Kushmerick, J. Lazorcik, C. H. Patterson, R. Shashidhar, D. S. Seferos, and G. C. Bazan, Nano Lett. 4, 639 (2004).

[22] C.R. Arroyo, T. Frederiksen, G. Rubio-Bollinger, M. Vélez, A. Arnau, D. Sánchez-Portal, and N. Agraï, Phys. Rev. B 81, 075405 (2010).

[23] L. A. Zotti, T. Kirchner, J. Cuevas, F. Pauly, T. Huhn, E. Scheer, and A. Erbe, Small 6, 1529 (2010).

[24] See Supplemental Material at http://link.aps.org/ supplemental/10.1103/PhysRevLett.109.226801 for a detailed explanation.

[25] Y. Kim, T. J. Hellmuth, D. Sysoiev, F. Pauly, T. Pietsch, J. Wolf, A. Erbe, T. Huhn, U. Groth, U. Steiner, and E. Scheer, Nano Lett. 12, 3736 (2012).
[26] J. M. Soler, E. Artacho, J. D. Gale, A. Garcia, J. Junquera, P. Ordejón, and D. Sanchez-Portal, J. Phys. Condens. Matter 14, 2745 (2002).

[27] M. Brandbyge, J. L. Mozos, P. Ordejón, J. Taylor, and K. Stokbro, Phys. Rev. B 65, 165401 (2002).

[28] J. P. Perdew, K. Burke, and M. Ernzerhof, Phys. Rev. Lett. 77, 3865 (1996).

[29] Y. Wang and H.-P. Cheng, Phys. Rev. B 86, 035444 (2012).

[30] S. Kümmel and L. Kronik, Rev. Mod. Phys. 80, 3 (2008).

[31] J. B. Neaton, M. S. Hybertsen, and S. G. Louie, Phys. Rev. Lett. 97, 216405 (2006).

[32] M. Paulsson and M. Brandbyge, Phys. Rev. B 76, 115117 (2007).

[33] M. Strange, C. Rostgaard, H. Häkkinen, and K.S. Thygesen, Phys. Rev. B 83, 115108 (2011).

[34] M. Strange, O. Lopez-Acevedo, and H. Häkkinen, J. Phys. Chem. Lett. 1, 1528 (2010).

[35] http://inelastica.sourceforge.net/.

[36] T. Frederiksen, M. Paulsson, M. Brandbyge, and A.-P. Jauho, Phys. Rev. B 75, 205413 (2007). 